ARTICLE

https://doi.org/10.1038/s41467-019-12012-z

\title{
Balancing hydrogen adsorption/desorption by orbital modulation for efficient hydrogen evolution catalysis
}

\author{
Feng Li (10 1, Gao-Feng Han (10 1, Hyuk-Jun Noh'1, Jong-Pil Jeon', Ishfaq Ahmad', Shanshan Chen²,3, \\ Changduk Yang (1) ${ }^{3}$, Yunfei Bu${ }^{4}$, Zhengping Fu ${ }^{5}$, Yalin Lu (i) ${ }^{5}$ \& Jong-Beom Baek (D) ${ }^{1}$
}

Hydrogen adsorption/desorption behavior plays a key role in hydrogen evolution reaction (HER) catalysis. The HER reaction rate is a trade-off between hydrogen adsorption and desorption on the catalyst surface. Herein, we report the rational balancing of hydrogen adsorption/desorption by orbital modulation using introduced environmental electronegative carbon/nitrogen $(\mathrm{C} / \mathrm{N})$ atoms. Theoretical calculations reveal that the empty $\mathrm{d}$ orbitals of iridium (Ir) sites can be reduced by interactions between the environmental electronegative $\mathrm{C} / \mathrm{N}$ and Ir atoms. This balances the hydrogen adsorption/desorption around the Ir sites, accelerating the related HER process. Remarkably, by anchoring a small amount of Ir nanoparticles (7.16 wt\%) in nitrogenated carbon matrixes, the resulting catalyst exhibits significantly enhanced HER performance. This includs the smallest reported overpotential at $10 \mathrm{~mA} \mathrm{~cm}^{-2}(4.5 \mathrm{mV})$, the highest mass activity at $10 \mathrm{mV}\left(1.12 \mathrm{~A} \mathrm{mg}_{\mathrm{lr}}{ }^{-1}\right)$ and turnover frequency at $25 \mathrm{mV}\left(4.21 \mathrm{H}_{2} \mathrm{~S}^{-1}\right)$ by far, outperforming Ir nanoparticles and commercial Pt/C.

\footnotetext{
${ }^{1}$ School of Energy and Chemical Engineering/Center for Dimension-Controllable Organic Frameworks, Ulsan National Institute of Science and Technology (UNIST), 50 UNIST, UIsan 44919, South Korea. ${ }^{2}$ MOE Key Laboratory of Low-grade Energy Utilization Technologies and Systems, CQU-NUS Renewable Energy Materials \& Devices Joint Laboratory, School of Energy \& Power Engineering, Chongqing University, Chongqing 400044, P. R. China. ${ }^{3}$ Department of Energy Engineering, School of Energy and Chemical Engineering, Perovtronics Research Center, Low Dimensional Carbon Materials Center, Ulsan National Institute of Science and Technology (UNIST), 50 UNIST, Ulsan 44919, South Korea. ${ }^{4}$ School of Environmental Science and Engineering, Nanjing University of Information Science and Technology, 219 Ningliu, Nanjing, Jiangsu 210044, P. R. China. ${ }^{5}$ CAS Key Laboratory of Materials for Energy Conversion, Hefei National Laboratory for Physical Sciences at Microscale, National Synchrotron Radiation Laboratory, University of Science and Technology of China, 96 Jinzhai, Hefei, Anhui 230026, P. R. China. Correspondence and requests for materials should be addressed to Y.B. (email: yunfei.bu@nuist.edu.cn) or to Z.F. (email: fuzp@ustc.edu.cn) or to J.-B.B. (email: jbbaek@unist.ac.kr)
} 
B ecause of its potential value in efficient and scalable hydrogen production, water splitting via the electrochemical hydrogen evolution reaction (HER) has attracted considerable attention from industrial and scientific communities $^{1,2}$. Even though they are the heart of hydrogen evolution catalysis, catalysts with satisfactory performance are still rare, despite the tremendous effort that has been devoted to improving HER catalysts ${ }^{3-11}$. Among various approaches, sulfur (S) vacancies and strain have been introduced to activate and optimize the basal plane of monolayer $2 \mathrm{H}-\mathrm{MoS}_{2}$, leading to the highest intrinsic HER activity among molybdenum-sulfide-based catalysts ${ }^{8}$. A high concentration of strained metallic $1 \mathrm{~T} \mathrm{WS} \mathrm{W}_{2}$ sites, obtained by chemical exfoliation, led to $\mathrm{WS}_{2}$ nanosheets with enhanced catalytic activity ${ }^{4}$. Ultrathin $\mathrm{Pt}$ nanowires on singlelayered $\mathrm{Ni}(\mathrm{OH})_{2}$ nanosheets with a unique hybrid nanostructure were also achieved, and exhibited unprecedented catalytic activity and stability toward $\mathrm{HER}^{6}$. Nonetheless, in spite of these achievements, strategic exploration to improve the HER performance of catalysts is still a great challenge.

From a fundamental viewpoint, the hydrogen adsorption/desorption behaviors of the solid surfaces of catalysts are of crucial importance in hydrogen evolution catalysis ${ }^{12,13}$. Stronger hydrogen adsorption enables faster proton supply for the reaction, however, on the other side, this leads to weaker hydrogen desorption and slower release of active sites. Too strong or too weak hydrogen adsorption/desorption will lead to either active site poisoning or inefficient proton supply, resulting in a sluggish hydrogen evolution reaction rate.

The hydrogen adsorption/desorption behaviors of transition metal-based catalysts depend largely on the interaction between the unfulfilled $d$ orbitals of the transition metals and the $s$ orbital of hydrogen ${ }^{14}$. As a result, hydrogen adsorption/desorption on such catalysts can be tailored by tuning the $d$ orbitals of the transition metals. With their small atomic radius and high electronegativity, the $p$ orbitals of carbon and nitrogen are able to efficiently interact with the $d$ orbitals of the transition metals ${ }^{15}$. This provides an important opportunity to enhance the catalytic activity of catalysts toward HER via orbital manipulation.

Herein, we demonstrate the rational balancing of hydrogen adsorption/desorption on a solid catalyst surface to archive significantly enhanced hydrogen evolution performance via orbital modulation. Density functional theory (DFT) calculations revealed that the empty $d$ orbitals of iridium (Ir) sites can be tailored by an electronegative carbon/nitrogen $(\mathrm{C} / \mathrm{N})$ environment, which will further balance hydrogen adsorption/ desorption behaviors and boost the hydrogen evolution catalysis on the surficial Ir sites. Importantly, even with a low-Ir concentration of $7.16 \mathrm{wt} \%$, when Ir nanoparticles (IrNP) were anchored on a hollow nitrogenated carbon matrix (IrHNC), the resulting catalyst exhibited HER performance superior to IrNP and commercial $\mathrm{Pt} / \mathrm{C}$, including much faster reaction kinetics, and much higher mass activity as well as intrinsic activity.

\section{Results}

DFT calculations. Figure 1 shows the different hydrogen adsorption/desorption behaviors on the solid surface of catalyst. For an efficient HER, fast reactant supply and fast product delivery are needing to be satisfied simultaneously, which required strong hydrogen adsorption and strong hydrogen desorption capabilities from the catalyst surface. However, as the two sides of the coin, they cannot be achieved at the same time. Thus, the hydrogen adsorption/desorption behaviors balancing offers an alternative way to enhance the HER performance of the catalysts.
With a weak hydrogen adsorption behavior, Ir was first selected for the hydrogen adsorption/ desorption behavior balancing ${ }^{16}$. As the thermally stable plane of Ir, the (111) plane is most stable and available in the experiment. Thus, the theoretical investigations were conducted on its thermally stable (111) plane. The structures of the calculation models for Ir without/with $\mathrm{C} / \mathrm{N}$ environment ( $\mathrm{Ir} / \mathrm{IrNC}$ ) are shown in Supplementary Figs. 1 and 2. The orbital profiles of Ir and IrNC were analyzed first. Figure 2 shows the different views of the orbitals above the Fermi level for Ir and IrNC, which are predominately composed of empty $d$ orbitals for Ir sites. The empty $d$ orbitals above the Fermi level in Fig. 2a, b, along with the projected density of states (DOS) distributions of the $\mathrm{Ir}, \mathrm{C}$, and $\mathrm{N}$ sites (Supplementary Fig. 3), reveal the strong interactions between the $\mathrm{Ir}$ and environmental $\mathrm{C} / \mathrm{N}$ sites. Because of a large wave function overlap, the $p$ orbitals of the $\mathrm{C} / \mathrm{N}$ sites directly interact with the $d_{z}^{2}$ orbitals of the nearby Ir sites. By introducing the $\mathrm{C} / \mathrm{N}$ environment, the empty $d$ orbitals of the surficial Ir sites for IrNC were remarkably reduced, benefiting the activation of surficial Ir sites and electron transport on the Ir surface of IrNC (Fig. 2c, d).

The orbital modulated electron structure of IrNC can be further illustrated using the electron density difference calculation, with Ir as the reference (Supplementary Fig. 4). The electrons of the surficial Ir sites on the $\operatorname{Ir}(111)$ surface are more like to transfer to nearby hollow fcc sites. Thus, the hollow fcc sites with high electron density are favored for hydrogen adsorption. Compared with Ir, the electron density of the surficial Ir sites of IrNC is much lower, while it is exactly the opposite for the hollow fcc sites. Therefore, the hollow fcc sites on the surface of IrNC with higher electron density will benefit hydrogen adsorption, leading to enhanced HER performance. Furthermore, the positive shift of the $d$-band center toward the Fermi level $\left(E_{\mathrm{F}}\right)$ in IrNC agrees well with the electron density difference results (Supplementary Fig. 5) ${ }^{17}$.

To obtain a direct understanding of the hydrogen adsorption/ desorption behaviors on the surface of $\mathrm{Ir}$ and IrNC, the calculation models were further configured with hydrogen adsorbates. Figure $3 \mathrm{a}, \mathrm{b}$ shows the optimized configurations and electron density differences of Ir and IrNC with hydrogen adsorbed, respectively. The projected DOS distributions of adsorbed hydrogen and surficial Ir were further analyzed (Fig. 3c and Supplementary Fig. 6). In contrast with Ir, stronger bonding of hydrogen on the surficial Ir sites was obtained for IrNC.

Further calculations were conducted to investigate the overall HER pathway on the surfaces of IrNC, taking Ir and $\mathrm{Pt}$ as references (Supplementary Fig. 7). $\mathrm{H}^{+}+\mathrm{e}^{-}$, adsorbed hydrogen $\left(\mathrm{H}^{*}\right)$ and $1 / 2 \mathrm{H}_{2}$ were considered as the initial, intermediate and final states of the reaction, respectively. The calculated free energy diagrams of the HER process on the surfaces of Ir, IrNC, and Pt are presented in Fig. 3d. Pt, which is known as the most efficient HER catalyst, exhibits a small upslope of $0.09 \mathrm{eV}$, revealing hydrogen desorption as the rate-determining step (RDS). Unlike Pt, weak hydrogen adsorption is the RDS of Ir, with a larger upslope of $0.25 \mathrm{eV}$. Significantly, the hydrogen adsorption/desorption behaviors of surficial Ir sites of the IrNC were largely balanced by the environmental $\mathrm{C} / \mathrm{N}$ sites, which introduced by orbital modulation, resulting in a much smaller upslope of $0.04 \mathrm{eV}$. Compared with $\mathrm{Pt}$, the interaction energy between hydrogen and IrNC surface would be not energetically favored for hydrogen adsorption, because of a little positive free energy change in the hydrogen adsorption process. However, this interaction energy would be more energetically favored for hydrogen desorption. The reaction rate of HER depends on the upslope process in the HER free energy diagram. With a much 

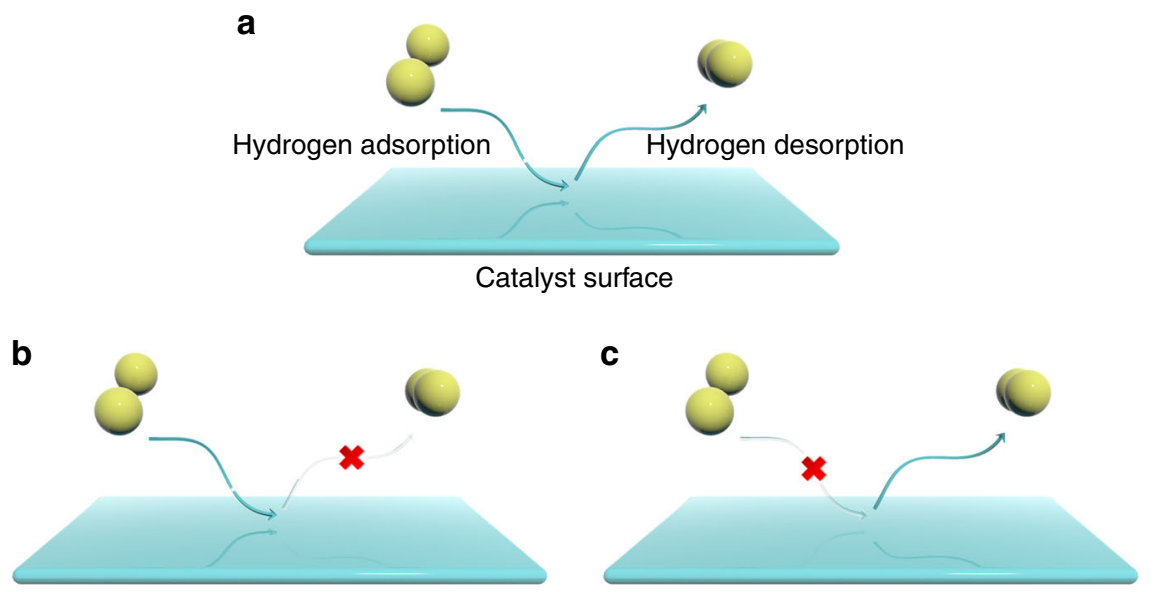

Fig. 1 Schematic illustration for the different hydrogen adsorption/desorption behaviors of catalyst surface. a Balanced hydrogen adsorption/desorption behavior. b, c Too strong and too weak hydrogen adsorptions, respectively, resulting in sluggish hydrogen evolution reaction rate. The dark yellow ball represents hydrogen atom

a

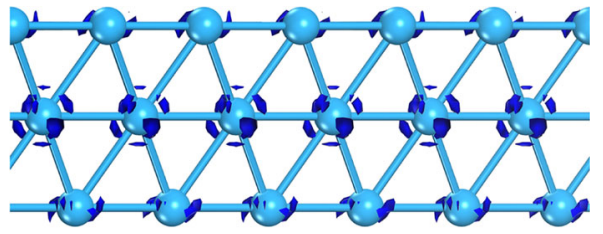

Ir $\quad \mathrm{C} \quad \mathrm{N}$

$\odot \odot O$

C

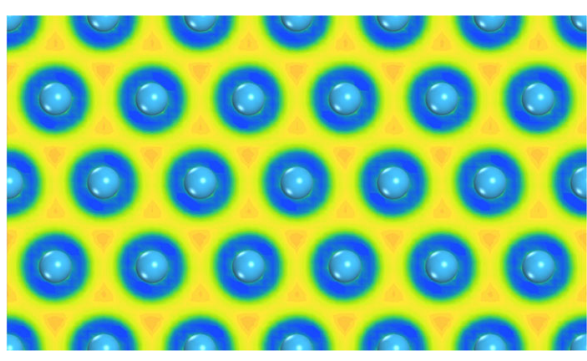

e



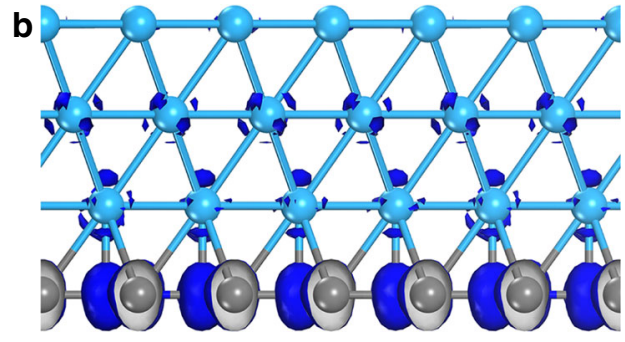

d

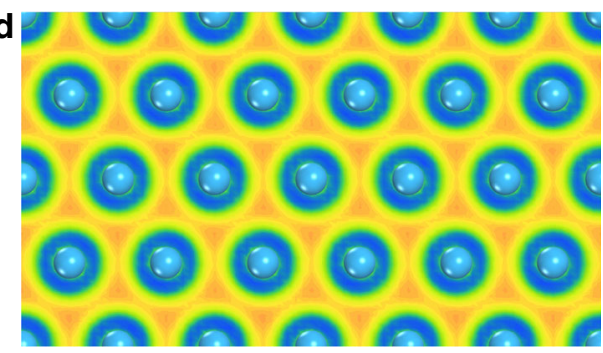

f

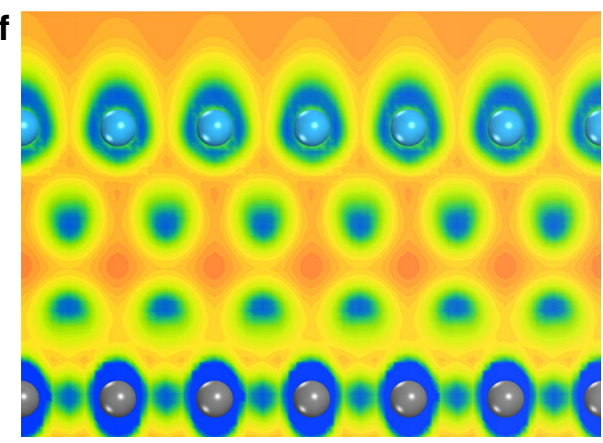

Fig. 2 Theoretical calculations of the orbitals for Ir and IrNC. a, c, $\mathbf{d}$ The isosurface and slices from the top/side-view of the orbitals above the Fermi level for Ir, respectively. $\mathbf{b}, \mathbf{d}, \mathbf{f}$ The isosurface and slices from the top/side-view of the orbitals above the Fermi level for IrNC, respectively

smaller upslope, the interaction energy between hydrogen and IrNC surface would be more favored by the hydrogen adsorption/desorption involved overall HER reaction process, accelerating the overall rate of HER reaction. The above results suggest that IrNC will exhibit strikingly improved catalytic activity toward the hydrogen evolution process, compared with Ir and Pt.
Catalyst characterizations. Inspired by the theoretical calculation results, hollow nitrogenated carbon nanospheres anchored with IrNP were prepared (IrHNC, Supplementary Figs. 8-10). The structure and crystallinity of the IrHNC were first investigated by field emission scanning electron microscope (Supplementary Fig. 11a) and high-power X-ray diffraction (HP-XRD, Supplementary Figs. $11 \mathrm{~b}$ and 12 ). The broad peak at around $23^{\circ}$ belongs 

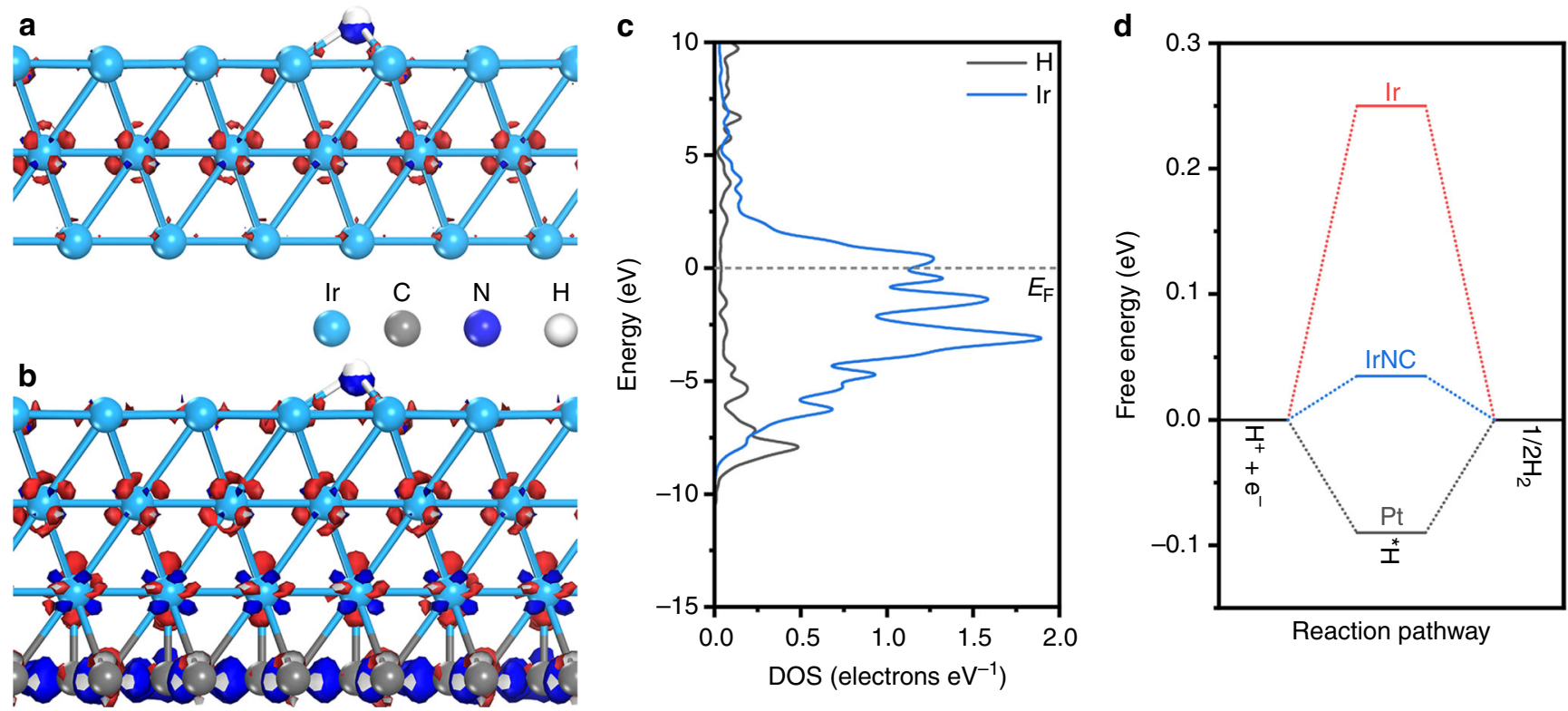

Fig. 3 Theoretical calculations of the hydrogen adsorption configured for Ir and IrNC. a, b The isosurfaces of the electron density difference for Ir and IrNC with $\mathrm{H}$ adsorption, respectively. c The projected density of states (DOS) distribution of adsorbed $\mathrm{H}$ and surficial Ir sites in IrNC, respectively. d The calculated free energy diagram of HER on the surface of Ir and IrNC at the equilibrium potential, respectively

to the carbon layer of the nitrogenated carbon with low crystallinity, while the weak peaks at around $40.6^{\circ}, 47.3^{\circ}$, and $69.1^{\circ}$ can be attributed to the (111), (200), and (220) planes of cubic Ir. Meanwhile, according to the Debye-Scherrer equation, an average particle size of IrNP is around $2.1 \mathrm{~nm}$.

The detailed morphology of the IrHNC was further studied on a high-resolution transmission electron microscope (HR-TEM, Fig. 4). The size of a building block in the IrHNC is around $700 \mathrm{~nm}$ (Fig. 4a). The hollow nanosphere had a porous structured shell with a thickness of about $14 \mathrm{~nm}$. The size distribution of IrNP was in the range of $0.9-2.5 \mathrm{~nm}$ and they were uniformly anchored in the porous shell (Fig. $4 \mathrm{~b}$ ). The lattice fringes in the HR-TEM image exhibited a $d$-spacing of $0.22 \mathrm{~nm}$, which can be assigned to the $\{111\}$ facets of cubic Ir (Fig. 4c). The homogeneous distribution of the $\mathrm{C}, \mathrm{N}$, and $\mathrm{Ir}$ elements was further revealed by the high-angle annular dark-field scanning transmission electron microscopy and element mapping images (Fig. 4d).

The analysis of the chemical composition of the IrHNC was conducted using X-ray photoelectron spectroscopy (XPS, Supplementary Fig. 13). The concentration of N species, mainly composed of pyridinic, graphitic, and oxidized $\mathrm{N}$ species, was around $4.45 \mathrm{at} \%{ }^{18}$. The peak at $61.4 \mathrm{eV}$ belongs to the metallic $\mathrm{Ir}^{\circ}$, which exhibited a low concentration of 0.36 at\% (Supplementary Table 1). Compared with the IrNPs, a slight blueshift was observed for IrHNC, which further suggests a strong interaction between the Ir and $\mathrm{N}$ species (Supplementary Figs. 14 and 15$)^{15}$. Highly sensitive X-ray absorption fine structure (XAFS) tests based on synchrotron radiation were performed to obtain deeper insight of the structural nature of the IrHNC. With $\mathrm{a}+4$ oxidation state, the position of the white line in the Ir $\mathrm{L}_{\mathrm{III}}$-edge $\mathrm{X}$-ray absorption near-edge structure (XANES) spectra of $\mathrm{IrO}_{2}$ was more positive than those of IrNP and IrHNC (Fig. 4e). Compared with the 0 oxidation state of IrNP, that of IrHNC exhibits a small positive shift, in agreement with the above XPS analysis. The white line features can be confirmed more clearly in the second derivatives of the Ir $\mathrm{L}_{\mathrm{III}}$-edge XANES spectra (Supplementary Fig. 16). The corresponding Fourier-transformed (FT) $\mathrm{k}^{3}$-weighted extended XAFS (EXAFSs) are shown in Fig. 4f. The main peaks in the EXAFS spectra of the IrNP and IrHNC at around $2.58 \AA$ belong to the $\mathrm{Ir}-\mathrm{Ir}$ coordination. Moreover, an $\mathrm{Ir}-\mathrm{N}$ coordination peak at around $1.59 \AA$ appears in the EXAFS spectrum of IrHNC, confirming the interaction between the Ir and $\mathrm{N}$ species.

A thermogravimetric analysis (TGA) further confirmed the Ir content of the IrHNC to be as low as $7.16 \mathrm{wt} \%$ (Supplementary Fig. 17). According to the nitrogen adsorption-desorption isotherms, the IrHNC possessed a specific surface area of $759.4 \mathrm{~m}^{2} \mathrm{~g}^{-1}$ and a pore volume of $0.76 \mathrm{~cm}^{3} \mathrm{~g}^{-1}$, respectively (Supplementary Fig. 18).

Catalyst performance. To verify the theoretical prediction, the catalytic HER performance of the obtained IrHNC was evaluated in nitrogen saturated $0.5 \mathrm{M}$ aq. $\mathrm{H}_{2} \mathrm{SO}_{4}$. IrNP and commercial Pt/ $\mathrm{C}$ were also investigated as references (Supplementary Figs. 19 and 20). Figure 5a shows the polarization curves of the IrHNC, IrNP, and Pt/C. Remarkably, with the same concentration of metal, IrHNC exhibited significantly enhanced HER performance. To achieve a current density of 10 and $100 \mathrm{~mA} \mathrm{~cm}^{-2}$, the IrHNC only needed small overpotentials of 4.5 and $39 \mathrm{mV}$, while those for IrNP and Pt/C are much larger (Fig. 5b). These values are also much smaller than those reported for efficient HER catalysts, revealing the superior catalytic activity of IrHNC (Supplementary Table 2) $6,19-22$.

The charge transfer resistance $\left(R_{\mathrm{ct}}\right)$ during the hydrogen evolution process was further studied by electrochemical impedance spectroscopy. At an overpotential of $10 \mathrm{mV}$, IrHNC exhibited the smallest $R_{\mathrm{ct}}$ of $4.2 \Omega$, which confirms that the fastest HER reaction kinetics occurred on the surface of the IrHNC (Fig. 5c, Supplementary Fig. 19c).

Mass activity and intrinsic activity are two important parameters to evaluate catalysts for potential practical applications. The mass activities of $\mathrm{IrHC}, \operatorname{IrNP}$, and $\mathrm{Pt} / \mathrm{C}$ at an overpotential of $10 \mathrm{mV}$ are $1.12,0.04$, and $0.25 \mathrm{~A} \mathrm{mg}_{\text {metal }}-1$, respectively (Fig. 5d). Remarkably, that value for IrHNC is 

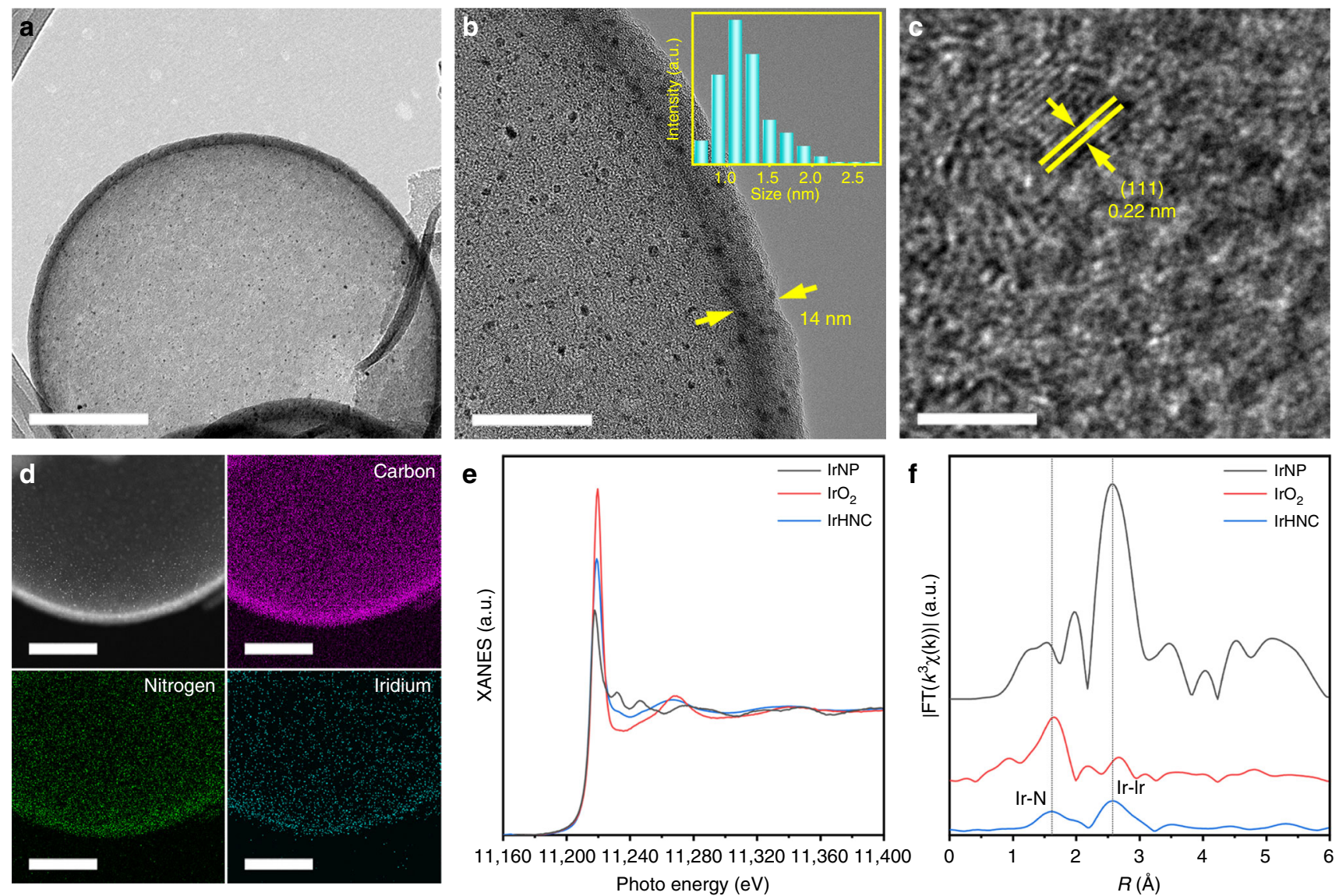

Fig. 4 Structural characterization of IrHNC. a, b Low-resolution TEM images of IrHNC. Inset is the corresponding particle size distribution of the Ir nanoparticles. c High-resolution TEM image of IrHNC. d Elemental mapping (purple: carbon, green: nitrogen, cyanic blue: iridium). e Ir $L_{111}$-edge XANES spectra of IrNP, IrO $\mathrm{I}_{2}$, and IrHNC, respectively. f Fourier transform (FT) of the Ir $\mathrm{L}_{111}$-edge EXAFS spectra of IrNP, IrO 2 , and IrHNC, respectively. Scale bars: a $200 \mathrm{~nm}, \mathbf{b} 40 \mathrm{~nm}, \mathbf{c} 3 \mathrm{~nm}, \mathbf{d} 100 \mathrm{~nm}$

over 28 and 4.5 times higher than those for IrNP and Pt/C, suggesting its higher potential for practical applications.

The turnover frequencies (TOFs) of the IrHNC and IrNP were further studied to obtain deeper insight into their intrinsic activities, using the reported method ${ }^{23}$. As shown in Fig. 5e, the TOFs of the IrHNC and IrNP were around 4.21 and $0.28 \mathrm{H}_{2} \mathrm{~s}^{-1}$ at $25 \mathrm{mV}$, respectively. Interestingly, the value for IrHNC is over 15 times higher than that for IrNP, indicating the IrHNC has significantly enhanced intrinsic activity. Moreover, this value is also far superior to other reported efficient HER electrocatalysts, including $\mathrm{Ru} @ \mathrm{C}_{2} \mathrm{~N}, \mathrm{RuCoP}, \mathrm{Fe} / \mathrm{GD}, \mathrm{CoN}_{x} / \mathrm{C}, \mathrm{Ni}-\mathrm{Mo}, \mathrm{Ni}_{2} \mathrm{P}$, and $\mathrm{Co}-\mathrm{NG}^{8,23-30}$. More importantly, the IrHNC also exhibited a Faradaic efficiency as high as about 100\% (Supplementary Fig. 19e).

The greatly improved reaction kinetics and intrinsic activity of IrHNC toward the hydrogen evolution catalysis are also consistent with the theoretical predictions.

\section{Discussion}

In summary, we report the rational design of an efficient catalyst for hydrogen evolution, by balancing hydrogen adsorption/desorption via orbital modulation. Theoretical calculations suggested that the $d$ orbitals of Ir sites can be manipulated through strong interactions with the $p$ orbitals of $\mathrm{C} / \mathrm{N}$ atoms. They suggested that orbital modulation would further balance the hydrogen adsorption/desorption behaviors of the surficial Ir sites, enabling efficient hydrogen evolution. Inspired by the theoretical results,
IrHNC was prepared by anchoring a low content of IrNP on nitrogenated carbon matrixes. As expected, the IrHNC exhibited significantly enhanced reaction kinetics, mass activity and intrinsic activity for the hydrogen evolution. This work not only highlights the rational design of a catalyst for efficient hydrogen evolution, but also introduces an opportunity to achieve enhanced catalytic performance for diverse reactions via orbital modulation.

\section{Methods}

Theoretical calculation. The first principle DFT calculations were performed using the Vienna Ab Initio Simulation Package. The Perdew-Burke-Ernzerhof (PBE) type gradient-corrected exchange-correlation potential can provide a better overall description of the electronic subsystem, which is especially suitable for the studies of molecular interaction with metal surfaces ${ }^{31}$. Meanwhile, van der Waals interactions have been usually considered for the combined system ${ }^{32}$. Thus, the PBE type gradient-corrected exchange-correlation potential was employed in this study, considering van der Waals interactions. Projector-augmented wave potentials were used to describe the ionic potentials. A cutoff energy of $400 \mathrm{eV}$ was used for the plane-wave basis set. A $4 \times 4 \times 1$ Monkhorst-Pack k-point setup was used and the atomic configurations were relaxed, with residual forces smaller than $0.01 \mathrm{eV} / \AA$. A vacuum thickness of around $15 \AA$ was set along the $\mathrm{z}$-direction to avoid interaction between periodic images ${ }^{33,34}$.

Synthesis of IrHNC. As a typical synthesis, 2-amino-2-(hydroxylmethyl)-1,3propanediol (Tris base, $0.169 \mathrm{~g})$ was dissolved in deionized water $(130 \mathrm{ml})$ with polystyrene (PS) microsphere $(750 \mathrm{~nm}, 0.5 \mathrm{~g})$, forming solution A. Dopamine hydrochloride $(0.5 \mathrm{~g})$ and $\mathrm{IrCl}_{3} \cdot \mathrm{xH}_{2} \mathrm{O}(15 \mathrm{mg})$ were dissolved in deionized water $(10 \mathrm{ml})$, forming solution $\mathrm{B}$. The above solutions were stirred for $1 \mathrm{~h}$ at ambient conditions. Solution B was quickly poured into solution A, and stirred for another $26 \mathrm{~h}$. The product was collected by centrifuge and dried in an oven under reduced 

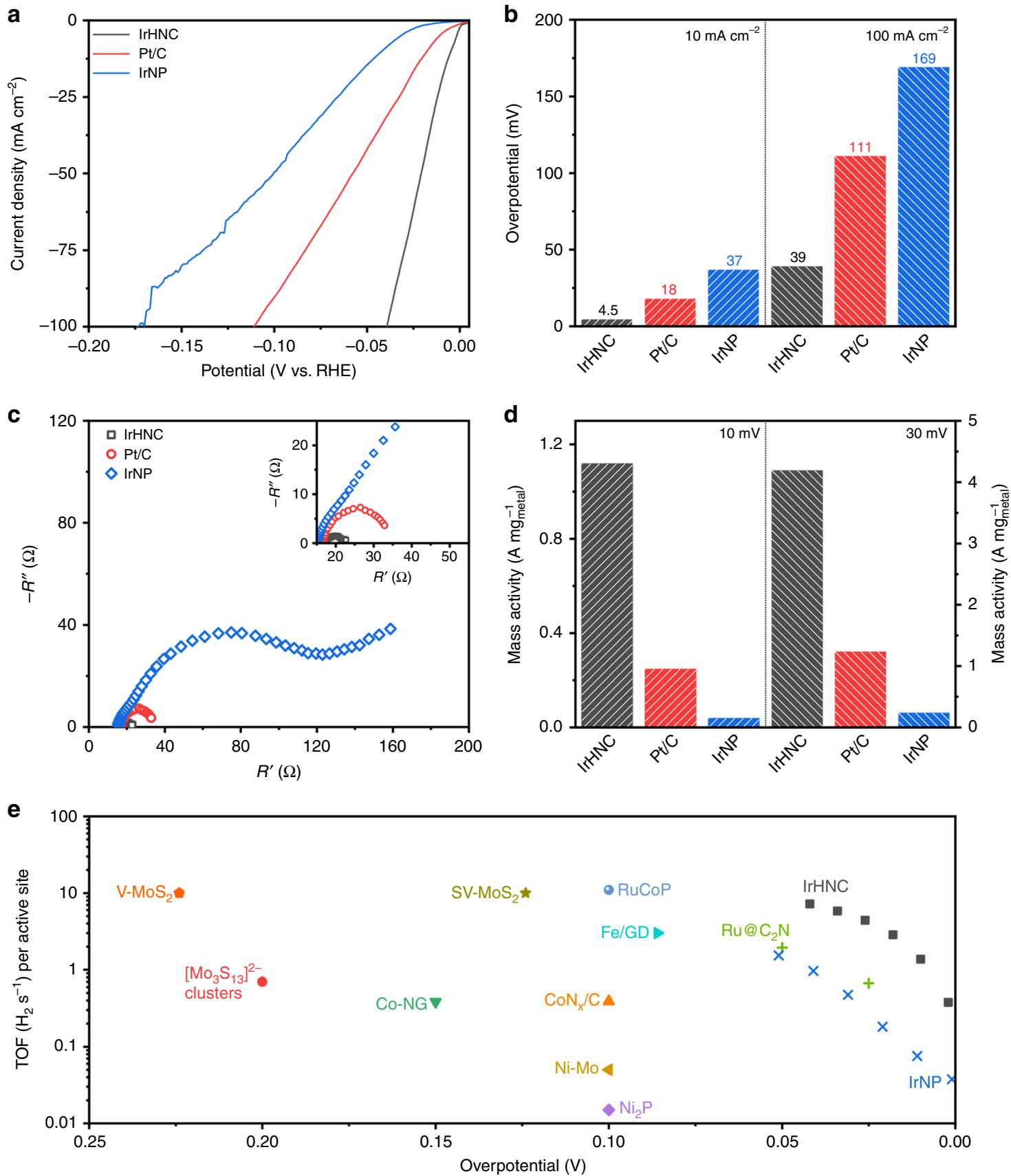

Fig. 5 Electrochemical performance of IrHNC. a Polarization curves IrHNC, Pt/C, and IrNP in nitrogen saturated $0.5 \mathrm{M}^{2}$ aq. $\mathrm{H}_{2} \mathrm{SO}_{4}$ solution. b Overpotentials of IrHNC, Pt/C and IrNP at current densities of 10 and $100 \mathrm{~mA} \mathrm{~cm}^{-2}$. c Electrochemical impedance spectroscopy (EIS) curves of IrHNC, Pt/C and IrNP at an overpotential of $10 \mathrm{mV}$. d Mass activities of IrHNC, Pt/C, and IrNP at overpotentials of 10 and $30 \mathrm{mV}$. e The TOF values of IrHNC and other recently reported HER electrocatalysts in acidic media. Scan rate: $5 \mathrm{mV} \mathrm{s}^{-1}$

pressure, then designated PS@IrPDA. The PS@IrPDA was annealed at $800^{\circ} \mathrm{C}$ for $2 \mathrm{~h}$ in an argon atmosphere, denoted as IrHNC. IrNP were prepared following the reported liquid phase reduction method $^{19}$, and designated IrNP.

Materials characterization. The morphologies of the samples were characterized on a field emission scanning electron microscope (SEM, Nanonova 230, FEI, USA) and high-resolution transmission electron microscopy (HR-TEM, JEM-2100F, JEOL, Japan). XRD patterns were obtained from a high-power X-ray diffractometer (D/MAZX 2500V/PC, Rigaku, Japan). The XPS test was conducted using an X-ray photoelectron spectrometer (K-alpha, Thermo Fisher Scientific, UK). The XAFS test was performed at the 6D UNIST-PAL beam-line of Pohang Light Source (PLSII) in Korea. The specific surface area was analyzed using nitrogen adsorption-desorption isotherms, and the Brunauer-Emmett-Teller (BET) method
(BELSORP-max, BEL, Japan). The TGA was conducted on a Thermogravimetric Analyzer (Q200, TA, USA).

Electrochemical characterizations. The electrocatalytic hydrogen evolution performance was evaluated using an electrochemical workstation (Ivium, Netherlands) with a typical three-electrode cell. To prevent $\mathrm{Pt}$ contamination, a graphite rod was used as the counter-electrode. $\mathrm{An} \mathrm{Ag} / \mathrm{AgCl}(3 \mathrm{M} \mathrm{KCl})$ electrode was used as a reference electrode. All the potentials were referenced vs. RHE. To form uniform catalyst inks, the catalysts and Nafion $5 \mathrm{wt} \%$ in a mixture of lower aliphatic alcohol and water, Aldrich Chemical Inc.) were dispersed in an isopropyl alcohol solution by ultrasonication. The inks were dropped onto a glassy carbon electrode $(3 \mathrm{~mm}$ in diameter) to form a film for the electrochemical tests. The loading amounts for IrHNC, IrNP, and $\mathrm{Pt} / \mathrm{C}$ were 18 and $18 \mu \mathrm{g}_{\mathrm{Pt}} \mathrm{cm}^{-2}$, respectively. Linear sweep voltammetry was conducted in a nitrogen saturated $0.5 \mathrm{M}$ aq. $\mathrm{H}_{2} \mathrm{SO}_{4}$ solution at a 
scan rate of $5 \mathrm{mV} \mathrm{s}^{-1}$. Electrochemical impedance spectroscopy measurements were conducted over a frequency range of $0.1-10,000 \mathrm{~Hz}$ at overpotentials of $10 \mathrm{mV}$. All the data presented were corrected with solution resistance.

\section{Data availability}

The data that supports the findings of this study are available from the corresponding author upon reasonable request.

Received: 2 June 2019 Accepted: 16 August 2019

Published online: 06 September 2019

\section{References}

1. Turner, J. A. Sustainable hydrogen production. Science 305, 972-974 (2004).

2. Norskov, J. K. \& Christensen, C. H. Chemistry-toward efficient hydrogen production at surfaces. Science 312, 1322-1323 (2006).

3. Cook, T. R. et al. Solar energy supply and storage for the legacy and non legacy worlds. Chem. Rev. 110, 6474-6502 (2010).

4. Voiry, D. et al. Enhanced catalytic activity in strained chemically exfoliated $\mathrm{WS}_{2}$ nanosheets for hydrogen evolution. Nat. Mater. 12, 850-855 (2013).

5. Morales-Guio, C. G., Stern, L. A. \& Hu, X. L. Nanostructured hydrotreating catalysts for electrochemical hydrogen evolution. Chem. Soc. Rev. 43, 6555-6569 (2014).

6. Yin, H. J. et al. Ultrathin platinum nanowires grown on single-layered nickel hydroxide with high hydrogen evolution activity. Nat. Commun. 6, 6430 (2015).

7. Zou, X. X. \& Zhang, Y. Noble metal-free hydrogen evolution catalysts for water splitting. Chem. Soc. Rev. 44, 5148-5180 (2015).

8. $\mathrm{Li}, \mathrm{H}$. et al. Activating and optimizing $\mathrm{MoS}_{2}$ basal planes for hydrogen evolution through the formation of strained sulphur vacancies. Nat. Mater. 15, 48-53 (2016).

9. Wang, X. S. et al. Strain effect in bimetallic electrocatalysts in the hydrogen evolution reaction. ACS Energy Lett. 3, 1198-1204 (2018)

10. Shan, J. Q. et al. Charge-redistribution-enhanced nanocrystalline Ru@IrO electrocatalysts for oxygen evolution in acidic media. Chemistry 5, 445-459 (2019).

11. Shan, J. Q., Ling, T., Davey, K., Zheng, Y. \& Qiao, S. Z. Transition-metaldoped RuIr bifunctional nanocrystals for overall water splitting in acidic environments. Adv. Mater. 31, 1900510 (2019).

12. Hinnemann, B. et al. Biomimetic hydrogen evolution: $\mathrm{MoS}_{2}$ nanoparticles as catalyst for hydrogen evolution. J. Am. Chem. Soc. 127, 5308-5309 (2005).

13. Subbaraman, R. et al. Enhancing hydrogen evolution activity in water splitting by tailoring $\mathrm{Li}^{+}-\mathrm{Ni}(\mathrm{OH})_{2}-\mathrm{Pt}$ interfaces. Science 334, 1256-1260 (2011).

14. Gong, M. et al. Nanoscale nickel oxide/nickel heterostructures for active hydrogen evolution electrocatalysis. Nat. Commun. 5, 4695 (2014).

15. Wu, Y. S. et al. Electron density modulation of $\mathrm{NiCo}_{2} \mathrm{~S}_{4}$ nanowires by nitrogen incorporation for highly efficient hydrogen evolution catalysis. Nat. Commun. 9, 1425 (2018).

16. Engstrom, J. R., Tsai, W. \& Weinberg, W. H. The chemisorption of hydrogen on the (111) and (110)-(1x2) surfaces of iridium and platinum. J. Chem. Phys. 87, 3104-3119 (1987).

17. Chen, Z. Y. et al. Tailoring the d-band centers enables $\mathrm{Co}_{4} \mathrm{~N}$ nanosheets to be highly active for hydrogen evolution catalysis. Angew. Chem. Int. Ed. 57, 5076-5080 (2018).

18. $\mathrm{Li}, \mathrm{F}$. et al. Boosting oxygen reduction catalysis with abundant copper single atom active sites. Energ. Environ. Sci. 11, 2263-2269 (2018).

19. Huang, X. et al. Solution-phase epitaxial growth of noble metal nanostructures on dispersible single-layer molybdenum disulfide nanosheets. Nat. Commun. 4, 1444 (2013).

20. Zhu, L. L. et al. A rhodium/silicon co-electrocatalyst design concept to surpass platinum hydrogen evolution activity at high overpotentials. Nat. Commun. 7, 12272 (2016)

21. Wang, P. T. et al. Precise tuning in platinum-nickel/nickel sulfide interface nanowires for synergistic hydrogen evolution catalysis. Nat. Commun. 8 , 14580 (2017).

22. Li, F. et al. Mechanochemically assisted synthesis of a Ru catalyst for hydrogen evolution with performance superior to Pt in both acidic and alkaline media. Adv. Mater. 30, 1803676 (2018).

23. Kibsgaard, J., Jaramillo, T. F. \& Besenbacher, F. Building an appropriate active-site motif into a hydrogen-evolution catalyst with thiomolybdate $\left[\mathrm{Mo}_{3} \mathrm{~S}_{13}\right]^{2-}$ clusters. Nat. Chem. 6, 248-253 (2014).

24. McKone, J. R., Sadtler, B. F., Werlang, C. A., Lewis, N. S. \& Gray, H. B. Ni-Mo nanopowders for efficient electrochemical hydrogen evolution. ACS Catal. 3, 166-169 (2013).
25. Popczun, E. J. et al. Nanostructured nickel phosphide as an electrocatalyst for the hydrogen evolution reaction. J. Am. Chem. Soc. 135, 9267-9270 (2013).

26. Fei, H. L. et al. Atomic cobalt on nitrogen-doped graphene for hydrogen generation. Nat. Commun. 6, 8668 (2015).

27. Liang, $\mathrm{H}$. W. et al. Molecular metal- $\mathrm{N}_{\mathrm{x}}$ centres in porous carbon for electrocatalytic hydrogen evolution. Nat. Commun. 6, 7992 (2015).

28. Mahmood, J. et al. An efficient and pH-universal ruthenium-based catalyst for the hydrogen evolution reaction. Nat. Nanotechnol. 12, 441-446 (2017).

29. $\mathrm{Xu}, \mathrm{J}$. Y. et al. Boosting the hydrogen evolution performance of ruthenium clusters through synergistic coupling with cobalt phosphide. Energy Environ. Sci. 11, 1819-1827 (2018).

30. Xue, Y. R. et al. Anchoring zero valence single atoms of nickel and iron on graphdiyne for hydrogen evolution. Nat. Commun. 9, 1460 (2018).

31. Perdew, J. P., Burke, K. \& Ernzerhof, M. Generalized gradient approximation made simple. Phys. Rev. Lett. 77, 3865-3868 (1996).

32. Tkatchenko, A. \& Scheffler, M. Accurate molecular van der Waals interactions from ground-state electron density and free-atom reference data. Phys. Rev. Lett. 102, 073005 (2009).

33. Hammer, B., Hansen, L. B. \& Norskov, J. K. Improved adsorption energetics within density-functional theory using revised Perdew-Burke-Ernzerhof functionals. Phys. Rev. B 59, 7413-7421 (1999).

34. Helveg, S. et al. Atomic-scale imaging of carbon nanofibre growth. Nature 427, 426-429 (2004).

\section{Acknowledgements}

This work was supported by the Creative Research Initiative (CRI, 2014R1A3A2069102), the Science Research Center (SRC, 2016R1A5A1009405), and the BK21 Plus (10Z20130011057) program through the National Research Foundation (NRF) of Korea. The authors wish to thank Pohang Accelerator Laboratory (Korea) for the synchrotron beam time.

\section{Author contributions}

J.-B.B. conceived the project and oversaw all the research phases. J.-B.B. and F.L designed the experiment. Y.L.L. and Z.P.F. carried out the theoretical studies. F.L. synthesized and characterized the samples. F.L. carried out the hydrogen experiments and data analysis. F.L., G.-F.H., Y.F.B., H.-J.N., J.-P.J., I.A., and S.S.C. were involved in the SEM, XRD, BET, TEM, and XAFS characterizations and data analysis. J.-B.B., F.L., C.Y., and Z.P.F. discussed the results. J.-B.B., F.L., and Z.P.F. wrote the paper. All the authors contributed to and commented on this paper.

\section{Additional information}

Supplementary Information accompanies this paper at https://doi.org/10.1038/s41467 019-12012-z.

Competing interests: The authors declare no competing interests.

Reprints and permission information is available online at http://npg.nature.com/ reprintsandpermissions/

Peer review information: Nature Communications would like to thank Shizhang Qiao and other, anonymous, reviewers for their contributions to the peer review of this work.

Publisher's note: Springer Nature remains neutral with regard to jurisdictional claims in published maps and institutional affiliations.

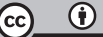

Open Access This article is licensed under a Creative Commons Attribution 4.0 International License, which permits use, sharing, adaptation, distribution and reproduction in any medium or format, as long as you give appropriate credit to the original author(s) and the source, provide a link to the Creative Commons license, and indicate if changes were made. The images or other third party material in this article are included in the article's Creative Commons license, unless indicated otherwise in a credit line to the material. If material is not included in the article's Creative Commons license and your intended use is not permitted by statutory regulation or exceeds the permitted use, you will need to obtain permission directly from the copyright holder. To view a copy of this license, visit http://creativecommons.org/ licenses/by/4.0/.

(C) The Author(s) 2019 